\title{
Suicide following discharge from in-patient psychiatric care
}

\author{
Mike J. Crawford
}

\begin{abstract}
The risk of suicide is higher during the period immediately following discharge from in-patient psychiatric care than at any other time in a service user's life. Demographic and clinical risk factors for suicide in this period are similar to those for suicide at other times and may not be specific enough to enable identification of those at greatest risk. Epidemiological studies suggest that factors related to service organisation and delivery (e.g. social support and continuity of care) are also important in the aetiology of suicide following hospital discharge. Interventions aimed at helping people in the period immediately following discharge from in-patient care have been developed. Although the effect of these interventions on suicidal behaviour has not been examined, available evidence suggests that efforts to increase patients' confidence and level of social support may be of value.
\end{abstract}

Suicide is of major public health importance in Britain and elsewhere. There are over 4500 deaths from suicide each year in the UK and reducing the rate of suicide remains a target for health care services in Britain and Ireland (World Health Organization, 2002). Recognition that some people are at much greater risk of suicide has prompted considerable efforts to develop and evaluate interventions aimed at preventing suicide in highrisk groups. These groups include individuals who present to services following an episode of deliberate self-harm (Hawton et al, 1998) and those attending primary care services with depression (Rutz et al, 1989). The rate of suicide is even higher in the first few weeks following discharge from psychiatric inpatient care, but interventions aimed at reducing suicide in this group have not been evaluated.

\section{Epidemiology}

There is evidence that the vast majority of people who die by suicide have a mental disorder at the time of their death, and up to a third have had recent contact with mental health services (Foster et al, 1997). For the latter group, the period of greatest risk for suicide is in the first few weeks following discharge from hospital. Data on over 2000 suicides among people in contact with mental health services from the National Confidential Inquiry into Suicide and Homicide reveal that a quarter of all deaths occur within the first 3 months of discharge from psychiatric hospital (Appleby et al, 1999b). It is estimated that almost $1 \%$ of those discharged from in-patient psychiatric care will kill themselves in the year following their discharge (Goldacre et al, 1993). Such deaths account for $10 \%$ of all suicides in the UK (Lewis et al, 1997). The rate of suicide is highest in the first few days after discharge from hospital, with $41 \%$ occurring before the first followup appointment (Appleby et al, 1999a).

The relationship between admission to hospital and suicide has been studied in greater detail among people with schizophrenia. Rossau \& Mortensen (1997) examined the characteristics of 508 people who were admitted to hospital for treatment of firstepisode schizophrenia in Denmark between 1970 and 1987. The rate of suicide was found to be highest in the 6 months following admission to hospital. It was higher following the first admission to hospital than following subsequent admissions and was also noted to be higher after discharge from general medical and surgical wards.

\section{Risk factors for suicide in the post-discharge period}

Basic demographic factors associated with suicide in the post-discharge period reflect risk factors for suicide in general. In the UK it is therefore more common among men and those with a diagnosis of depression and other affective disorders (Goldacre et al, 1993; Geddes et al, 1997). Case-control studies

Mike Crawford is a senior lecturer in psychiatry in the Department of Psychological Medicine at Imperial College London (Claybrook Centre, Claybrook Road, London, W6 8LN, UK. Tel: 0207386 1233; fax: 0207386 1216; e-mail: m.crawford@imperial.ac.uk). He specialises in mental health services research and is involved in the development and evaluation of complex psychosocial interventions. 
have been used to examine risk factors for suicide in the post-discharge period in greater detail. Appleby et al (1999a) compared the characteristics of 149 people who died by suicide within 5 years of discharge from psychiatric wards in Greater Manchester with 149 control patients. Those who died by suicide were more likely to have had a past history of deliberate self-harm, to have communicated ideas of suicide following their discharge from hospital and to have had a decrease in the level of care agreed at the last appointment before death. Other statistically significant associations with completed suicide were not found. This may have been in part due to the relatively small size of the study sample.

A larger study was subsequently conducted in south-west England by King et al (2001). Clinical records of 234 patients who had died by suicide within 1 year of hospital discharge were examined and the power of the study was further increased by recruiting two control patients for each of these cases. A range of clinical and service-related factors were found to be associated with an increased risk of suicide. These, together with factors identified in other studies, are summarised in Box 1.

\section{Why is the rate of suicide higher in the post-discharge period?}

Although social and demographic factors associated with suicide in the post-discharge period give an indication of those at greatest risk, they do not provide a basis for explaining why the rate of suicide is higher at this time than at other points in a person's life. Of other factors examined by King et al (2001), the strongest association with suicide was having a keyworker on holiday or about to leave the service at the time of the incident. This resulted in a six-fold increase in the likelihood of suicide. In discussing the findings of the study the authors acknowledged that this unexpectedly strong association might have been affected by the retrospective nature of the study, with bias in recording clinical events with hindsight. None the less, this, together with the association with change of consultant at the time of admission, gives rise to the possibility that reduced continuity of care is implicated in suicide following discharge from inpatient care. A variety of other possible explanations have been suggested for the excess of suicides in the post-discharge period. These include changes in the levels of insight that patients experience and reduced social support.

There is ample evidence to suggest that suicidal ideation is more frequent and the degree of suicidal intent higher among people with schizophrenia who have good insight into their illness (Schwartz,
Box 1 Factors associated with increased risk of suicide in the post-discharge period

Social and demographic factors

- Gender: male

- Age: over 45

- Unemployment

- Social isolation: living alone

- Ethnicity: non-White

Clinical factors

- First admission to hospital

- Suicidal ideation at time of admission

- Diagnosis: depression and affective psychosis

- Past history of deliberate self-harm

- Mental state: hopelessness

Service organisation and delivery

- 'Unplanned' discharge

- Key personnel on leave or leaving

- Short admissions (under 7 days)

- Admitted under a 'new' consultant

- Time when not in contact with services in the period following discharge from hospital

2000). Attempted suicide is also more common among those who show greater awareness of their symptoms and social circumstances (Amador et al, 1996). It therefore seems likely that the reduction in symptoms and the increased insight that occur after a period of in-patient treatment contribute to higher levels of suicide in the post-discharge period.

Prospective studies have repeatedly demonstrated that mental illness has a negative effect on the extent and quality of social support that people experience (Borge et al, 1999). Those who have been admitted to a psychiatric hospital do not appear to have less social support than those who have mental health problems but have not been admitted (Dickinson et al, 2002). However, surveys of people who have been discharged from a psychiatric hospital suggest that many find the reduced social support they experience after leaving hospital distressing. Simons et al (2002) conducted a survey of 173 people discharged from psychiatric hospitals in eight Scottish regions. The authors commented that, although patients who had had previous admissions to hospital generally felt prepared for their discharge, some people, especially those admitted for the first time, felt anxious about the prospect of going back to their homes. Analysis of qualitative data from respondents highlighted the value that many service users placed on having periods of leave away from the ward. Several respondents reported that such periods had enabled them to build up their confidence and helped to 'ease them back' into life at home. 


\section{Interventions to improve patient care around the time of discharge}

Concerns about arrangements for discharging patients from psychiatric wards have been expressed for some time. In addition to the increased level of suicide during this period, patients are more likely to drop out of contact with services at this time and levels of readmission to hospital are also high. It is reported that as many as a quarter of patients are readmitted during the 3 months after their discharge from in-patient psychiatric care (Boydell et al, 1991).

Problems in coordinating care between in-patient and community services have been highlighted as an impediment to discharge planning, and decreases in the number of in-patient places may result in pressure to discharge others before follow-up arrangements have been finalised (Durgahee, 1996). These observations are supported by the finding that wards where patient turnover is higher are those with the highest rate of readmission (Heggestad, 2001).

A variety of different interventions have been suggested for trying to help patients in the period around their discharge from hospital. These are summarised in Table 1. Little is known about the impact of these interventions, and their effect, if any, on the likelihood of suicide in the post-discharge period is unknown (Crawford et al, 2004). However, available evidence suggests that discharge preparation groups, meeting out-patient staff before discharge and the efforts of discharge coordinators lead to increased patient attendance at follow-up appointments. Several of these interventions aim to improve continuity of care and increase the amount of social support that patients experience following their discharge from hospital. Associations between suicide in the post-discharge period and poor social support and decreased continuity of care raise the possibility that such interventions could also reduce the likelihood of suicide following discharge from hospital, but evidence to support this hypothesis does not exist.

Clinical trials with sufficient power to examine the impact of interventions on suicide following discharge from in-patient units are unlikely to be conducted in the near future. Such studies would need to include thousands of participants in order to demonstrate clinically significant reductions in the rate of suicide. Although the epidemiology of fatal and non-fatal deliberate self-harm differs,

\section{Table 1 Interventions aimed at supporting patients during the discharge period}

Intervention

Prior to discharge

Periods of trial leave

Discharge preparation groups

Around the discharge period Discharge coordinators (transitional nurses)

Meeting out-patient staff

Support from 'buddies' (trained service users)

Following discharge Day hospital

Early out-patient follow-up
Aims

To help patients feel better able to cope in their own homes To assess the impact of discharge on patients' health and ability to cope To identify problems that may occur following discharge

To provide patients with information about problems that might be encountered during the post-discharge period and sources of help and support at that time

To enable patients to hear about and learn from the experiences of other service users

To facilitate communication between hospital, community and primary care services

To facilitate liaison with carers

To help ensure that patients are aware of follow-up arrangements and crisis services

To ensure that patients are aware of follow-up arrangements To encourage patients to attend follow-up appointments and other related services

To improve social support

To help patients cope with practical and emotional problems experienced following hospital discharge

To increase social support

To monitor mental state

To help patients cope with practical and emotional problems

To monitor mental state during a vulnerable period

To increase attendance at follow-up appointments 
suicidal ideation and behaviour may provide a guide to the impact of interventions on completed suicide and should therefore be included in future evaluations of interventions aimed at supporting patients during the post-discharge period. In the meantime, recommendations for service provision in the postdischarge period have already been made. The findings of the Confidential Inquiry into Suicide and Homicide by People with Mental Illness led to the recommendation that all patients with severe mental illness or a history of deliberate self-harm within the previous 3 months should be followed up within a week of their discharge (Department of Health, 2001). Ensuring that patients have access to follow-up services in the period immediately following their discharge from hospital makes good sense. Deciding who should receive this and other interventions is a more difficult task.

\section{Who should be targeted?}

Although the characteristics of patients most likely to take their own lives during the post-discharge period have been established, the strength of the association between these risk factors and suicide is not sufficient to allow accurate prediction of those who will kill themselves. For instance, of the 134 suicides among 14240 people discharged from hospitals in Oxfordshire reported by Goldacre et al (1993), only a third had a diagnosis of severe mental illness. Although men are at greater risk of suicide in the post-discharge period, over $40 \%$ of suicides in this study were by women. Risk assessment is now a part of routine clinical practice in many areas, but the assessment of risk does not enable mental health care professionals to predict the future (Crawford, 2003). Rather than trying to identify patients at greatest risk of suicide, these findings imply that efforts need to be made to enhance support and follow-up of all patients discharged from hospital.

\section{Conclusions}

Increased risk of suicide among people who have contact with mental health services is accentuated in the period immediately following discharge from in-patient care. Risk factors for suicide at this time have been described but do not provide a basis for targeting interventions aimed at suicide prevention. Higher rates of suicide following discharge from general medical wards provide further evidence that the transition from in-patient care to home is a stressful one. Interventions aimed at enhancing continuity of care and increasing social support have been developed but their impact on suicidal ideation and behaviour has not been examined. In the absence of such evidence, helping patients prepare for discharge by providing them with clear information about follow-up plans and crisis services and helping them readjust to life at home through periods of leave from the ward would seem worthwhile.

\section{References}

Amador, X. F., Friedman, J. H., Kasapis, C., et al (1996) Suicidal behavior in schizophrenia and its relationship to awareness of illness. American Journal of Psychiatry, 153, 1185-1188.

Appleby, L., Dennehy, J. A., Thomas, C. S., et al (1999a) Aftercare and clinical characteristics of people with mental illness who commit suicide: a case-control study. Lancet, 353, 1397-1400.

Appleby, L., Shaw, J., Amos, T., et al (1999b) Suicide within 12 months of contact with mental health services: National Clinical Survey. BMJ, 318, 1235-1239.

Borge, L., Martinsen, E. W., Ruud, T., et al (1999) Quality of life, loneliness, and social contact among long-term psychiatric patients. Psychiatric Services, 50, 81-84.

Boydell, K., Malcolmson, S. \& Sikerbol, K. (1991) Early rehospitalization. Canadian Journal of Psychiatry, 36, 743745 .

Crawford, M. J. (2003) Delivering safer services: can suicide and homicide among people in contact with mental health services be predicted? Expert Review of Neurotherapeutics, 3, 575-580.

Crawford, M. J., De Jonge, E., Freeman, G. K., et al (2004) Providing continuity of care for people with severe mental illness: a narrative review. Social Psychiatry and Psychiatric Epidemiology, 39, 265-272.

Department of Health (2001) Safety First: Five Year Report of The Confidential Inquiry into Suicide and Homicide by People with Mental Illness. London: Department of Health.

Dickinson, D., Green, G., Hayes, C., et al (2002) Social network and social support characteristics amongst individuals recently discharged from acute psychiatric units. Journal of Psychiatric Mental Health Nursing, 9, 183-189.

Durgahee, T. (1996) Discharge of psychiatric patients into the community: how many more must die? British Journal of Nursing, 5, 618-621.

Foster, T., Gillespie, K. \& McClelland, R. (1997) Mental disorders and suicide in Northern Ireland. British Journal of Psychiatry, 170, 447-452.

Geddes, J. R., Juszczak, E., O’Brien, F., et al (1997) Suicide in the 12 months after discharge from psychiatric in-patient care, Scotland 1968-92. Journal of Epidemiology and Community Health, 51, 430-434.

Goldacre, M., Seagroatt, V. \& Hawton, K. (1993) Suicide after discharge from psychiatric in-patient care. Lancet, 342, 283-286.

Hawton, K., Arensman, E., Townsend, E., et al (1998) Deliberate self-harm: systematic review of efficacy of psychosocial and pharmacological treatments in preventing repetition. BMJ, 317, 441-447.

Heggestad, T. (2001) Operating conditions of psychiatric hospitals and early readmission - effects of high patient turnover. Acta Psychiatrica Scandinavica, 103, 196-202.

King, E. A., Baldwin, D. S., Sinclair, J. M., et al (2001) The Wessex Recent In-Patient Suicide Study, 1. Case-control study of 234 recently discharged psychiatric patient suicides. British Journal of Psychiatry, 178, 531-536.

Lewis, G., Hawton, K. \& Jones, P. (1997) Strategies for preventing suicide. British Journal of Psychiatry, 171, 351354.

Rossau, C. D. \& Mortensen, P. B. (1997) Risk factors for suicide in patients with schizophrenia: nested case-control study. British Journal of Psychiatry, 171, 355-359.

Rutz, W., Walinder, J., Eberhard, G., et al (1989) An educational program on depressive disorders for general practitioners on Gotland: background and evaluation. Acta Psychiatrica Scandinavica, 79, 19-26. 
Schwartz, R. C. (2000) Insight and suicidality in schizophrenia: a replication study. Journal of Nervous and Mental Disorders, 188, 235-237.

Simons, L., Petch, A. \& Caplan, R. (2002) Don't They Call It Seamless Care? A Study of Acute Psychiatric Discharge. Edinburgh: Scottish Executive Social Research.

World Health Organization (2002) Suicide Prevention in Europe: The WHO European Monitoring Survey on National Suicide Prevention Programmes and Strategies. Copenhagen: WHO Regional Office for Europe.

\section{MCQs}

1 The likelihood of suicide in the post-discharge period is higher among:

a men than women

$b$ those under 45 years of age

c those with a diagnosis of depression

d those with a past history of deliberate self-harm

e British White compared with ethnic minority service users.

2 The rate of suicide among people with schizophrenia is increased:

a following discharge from general medical wards

b following discharge after a first admission compared with subsequent ones

c among those admitted to hospital on a compulsory basis

d among those who are socially isolated

e in the first week following discharge from hospital compared with the following 3 weeks.

3 The National Confidential Inquiry into Suicide and Homicide by People with Mental Illness:

a reported that suicide was associated with discharge from hospital after short admission (less than 7 days) b reported that most incidents of suicide following discharge from hospital occurred before the first follow-up appointment

c only covers suicides that occur in England and Wales

d recommended that all people with severe mental illness receive a follow-up appointment within a week of their discharge from hospital

e recommended that all people with a history of deliberate self-harm in the previous 3 months receive a follow-up appointment within a week of their discharge from hospital.

4 Clinical trials suggest that the following interventions increase patient attendance at follow-up appointments following hospital discharge:

a periods of trial leave from the ward

b referral to a day hospital

c attendance at structured programme of discharge preparation groups

d meeting out-patient staff prior to discharge

e early out-patient follow-up.

5 With regard to social support:

a suicide in the post-discharge period is more common among those with poor social support

$\mathrm{b}$ suicide in the post-discharge period is less common among those discharged to shared accommodation

c among people in contact with mental health services, admission to hospital leads to a subsequent decrease in the amount of social support they experience

d discharge coordinators aim to improve social support by meeting patients regularly in the weeks following their discharge from hospital

e peer support has been used to try to enhance social support in the period before and after discharge from hospital. 